\title{
The Differentiation of Bordetella parapertussis and Bordetella bronchiseptica from Humans and Animals as Determined by DNA Polymorphism Mediated by Two Different Insertion Sequence Elements Suggests Their Phylogenetic Relationship
}

\author{
ANNEKE VAN DER ZEE, ${ }^{*}$ HELEEN GROENENDIJK, ${ }^{2}$ MARCEL PEETERS, ${ }^{1}$ AND FRITS R. MOOI ${ }^{2}$ \\ Laboratory of Medical Microbiology, St. Elisabeth Hospital, 5000 AS Tilburg, ${ }^{1}$ and Laboratory for Infectious Diseases \\ Research, National Institute of Public Health and Environment, 3720 BA Bilthoven, ${ }^{2}$ The Netherlands
}

\begin{abstract}
We describe a novel insertion sequence (IS) element, IS1002, which was found to be closely related to IS481, which is found only in Bordetella pertussis; we found that these two IS elements have a level of sequence identity of $61.5 \%$ and also have almost identical terminal inverted repeats. IS1002 was present in both $B$. pertussis and Bordetella parapertussis strains isolated from humans. In contrast, IS1002 was absent from B. parapertussis strains isolated from sheep. A DNA fingerprint analysis performed with another IS element, IS1001, which is present in B. parapertussis and Bordetella bronchiseptica, revealed that $B$. parapertussis isolates obtained from sheep are distinct from human isolates. Thus, human and ovine $B$. parapertussis strains comprise two distinct populations, indicating that little or no transmission occurs between sheep and humans. An IS-associated restriction fragment length polymorphism analysis revealed that $B$. parapertussis strains isolated from sheep are genetically more polymorphic than the human $B$. parapertussis population, which is genetically very homogeneous. This suggests that human $B$. parapertussis strains diverged from a single clone only recently. IS1001 is present in a subset of $B$. bronchiseptica strains that were derived mainly from pigs and rabbits, suggesting that these strains had a common ancestry. On the basis of the results of a comparison of IS1001 band patterns and IS1001 sequences, ovine and human $B$. parapertussis strains appear to have evolved independently from $B$. bronchiseptica strains and to have adapted to different hosts (sheep and humans). Once in the human host, $B$. parapertussis probably acquired IS1002 from B. pertussis. In contrast to human $B$. parapertussis isolates, $B$. pertussis strains produced polymorphic IS1002-related DNA fingerprint patterns.
\end{abstract}

Bordetella bronchiseptica, Bordetella parapertussis, and Bordetella pertussis are pathogens of respiratory tracts and have adapted to a variety of mammalian hosts $(6,25)$. B. bronchiseptica is found in a wide range of animals, but only rarely in humans. Conversely, $B$. pertussis is an obligate human pathogen, causing whooping cough or pertussis. Although it has been assumed that $B$. parapertussis is also a strictly human pathogen (12), this organism has recently been isolated from both healthy sheep and sheep affected with chronic nonprogressive pneumonia $(5,8,24)$.

It has been estimated that between 5 and $30 \%$ of all pertussis cases are caused by $B$. parapertussis $(16,20)$. However, the incidence of $B$. parapertussis infections may be underestimated because this bacterium causes less severe symptoms than $B$. pertussis and infections may therefore go unnoticed (16). Indeed, serological surveys have shown that between 40 and $90 \%$ of adults have antibodies against $B$. parapertussis, indicating that most humans are infected by $B$. parapertussis during their lifetimes $(10,21)$. Although $B$. parapertussis generally causes a milder disease than $B$. pertussis, there have been numerous examples of severe, life-threatening infections caused by $B$. parapertussis $(11,30)$.

Multilocus enzyme electrophoresis (MEE) analysis has revealed that the genetic diversity of human $B$. parapertussis and $B$. pertussis strains is very limited, suggesting that these organisms descended from a common ancestor recently (23). $B$.

\footnotetext{
* Corresponding author. Mailing address: Laboratory for Infectious Diseases Research, National Institute of Public Health and Environment, P.O. Box 1, 3720 BA Bilthoven, The Netherlands. Phone: 3130-274.2113. Fax: 31-30-274.4414. Electronic mail address: fr.mooi (a)rivm.nl.
}

bronchiseptica strains are more polymorphic than human $B$. parapertussis and $B$. pertussis strains, indicating that $B$. bronchiseptica represents an older lineage. Notwithstanding these differences, $B$. bronchiseptica, B. parapertussis, and B. pertussis are very closely related and may be considered members of a single species (23). It has been suggested that human $B$. parapertussis and $B$. pertussis are merely clones of $B$. bronchiseptica which have become adapted to human hosts (23).

Given the observation that $B$. parapertussis is found both in sheep and in humans, one may raise the question whether sheep constitute a reservoir from which transmission of $B$. parapertussis to humans (or vice versa) takes place. If no such transmission occurs, one may expect the human and sheep strains to be distinct. Preliminary support for such a distinction was found by Porter et al. (24), who found that $B$. parapertussis strains isolated from sheep and humans can be differentiated by biochemical characteristics and MEE data.

To characterize the putative differences between $B$. parapertussis strains isolated from sheep and humans in more detail and to determine the phylogenetic relationships among the three Bordetella species, we studied the DNA polymorphism associated with repetitive DNA sequences present in the Bordetella species.

The following three repetitive DNA elements have been found in the genus Bordetella previously: two insertion sequence (IS) elements, IS481 (19) and IS1001 (27), and one uncharacterized element (19). IS 481 is found exclusively in $B$. pertussis, and $B$. pertussis strains contain approximately 100 copies per genome. van der Zee et al. (27) found IS1001 in $B$. parapertussis and in some, but not all, $B$. bronchiseptica strains and did not find IS1001 in B. pertussis. In the study of van der Zee et al. only Dutch $B$. parapertussis strains isolated from 
humans were investigated, and no IS1001-associated restriction fragment length polymorphism (RFLP) was observed. This is in contrast to B. bronchiseptica, in which different RFLP patterns were found (27).

The uncharacterized repetitive element was originally identified in $B$. pertussis, and this element was found to be located adjacent to a copy of IS481 (19). It was also found to be present in $B$. parapertussis strains isolated from humans (19). In order to use this repetitive DNA element for strain differentiation and phylogenetic analysis, we characterized it and showed that it is an IS element. Furthermore, in this paper we describe the distribution of and RFLP associated with this IS element and with IS1001 in $B$. parapertussis strains obtained from both humans and sheep and in $B$. bronchiseptica strains isolated from humans and a variety of different animals. The phylogenetic relationships among the three Bordetella species are discussed below.

\section{MATERIALS AND METHODS}

Bacterial strains and media. The origins of the isolates used in this study are shown in Table 1 . We used 103 B. pertussis strains and 31 B. parapertussis strains isolated from humans with clinical symptoms of whooping cough. B. parapertussis strains were kindly provided by J. Mertsola (Turku, Finland) (five strains), C. H. Wirsing von König (Krefeld, Germany) (three strains), P. Short (Porirua, New Zealand) (three strains), J. Porter, (Edinburgh, United Kingdom) (two strains), and T. Aoyama (Kawasaki, Japan) (four strains). A total of 17 B. parapertussis strains isolated from sheep were provided by J. Porter, and 5 B. parapertussis strains isolated from sheep were provided by B. W. Manktelow (Palmerston North, New Zealand). The 41 B. bronchiseptica strains which we studied were isolated from various animals having different geographic origins, and 30 of these strains were provided by W. Gaastra, (Utrecht, The Netherlands). All of the Bordetella strains were grown on Bordet-Gengou agar plates.

DNA techniques. Preparation of chromosomal DNA and isolation of plasmid DNA were carried out as described previously (3). Restriction endonucleases and T4 ligase were used as recommended by the manufacturer. Cloning procedures were performed by using pEMBL19 and Escherichia coli DH5 $\alpha$ (9).

Southern blotting. For the IS1001- and IS1002-based fingerprint analyses, chromosomal DNAs were digested with $S p h I$ and SmaI, respectively. DNA fragments were separated by agarose gel electrophoresis on $0.8 \%$ agarose gels in TAE buffer at $4 \mathrm{~V} / \mathrm{cm}$ for $5 \mathrm{~h}$ and blotted onto Hybond $\mathrm{N}+$ membranes (Amersham, Buckinghamshire, United Kingdom). Southern blotting was performed as described by Maniatis et al. (18). The IS1001 probe that was used in this study was the 498-bp PCR fragment generated by primer A (5'-CGCCGCTTGATG ACCTTGATA-3') and primer Z (5'-CACCGCCTACGAGTTGGAGAT-3'), as described previously (27). This internal IS 1001 fragment does not contain restriction recognition sites for $S p h \mathrm{I}$ or $S m a \mathrm{I}$. The IS 1002 probe (Fig. 1, probe 2) was 293 bp long and was generated by PCR by using primers HG1 ( $5^{\prime}$-GCCGAT GCGTTCCATATA-3') and HG2 (5'-AGCCCTTCTTGTTAACAGGG-3'), corresponding to positions 424 to 406 and 131 to 151 on the sequence of IS1002a, respectively (Fig. 2). PCR fragments were isolated from gels by using Geneclean (Bio 101, La Jolla, Calif.) and were labeled with peroxidase by using the method recommended by the manufacturer (ECL, Amersham, United Kingdom). The hybridization conditions used when IS1001 was the probe were the conditions recommended in the ECL kit. Hybridization of B. pertussis strains with IS 1002 was performed under higher-stringency conditions by lowering the $\mathrm{NaCl}$ concentration in the hybridization buffer from 0.5 to $0.1 \mathrm{M}$. This was done to minimize hybridization to IS481, whose sequence is similar to the IS $1002 \mathrm{se}$ quence.

Cloning of IS 1002 from B. parapertussis and B. pertussis. IS1002 was isolated from $B$. parapertussis B24 (a human strain isolated in The Netherlands) by randomly cloning PstI fragments. Recombinant clones were screened with the HpaI-ClaI fragment on cosmid p11-11 (Fig. 1, probe 1), which contained repetitive DNA derived from $B$. pertussis. One hybridizing plasmid, designated pRPP30, was selected, and this plasmid contained a 1.8-kb Pst I fragment. Inverse PCR with primers H (5'-GGCCTGCCTCATCGTCCAGTT- $\left.3^{\prime}\right)$ and C (5'ATCGGGCCCAGAAACCGCAA-3' ), corresponding to positions 187 to 166 and 556 to 575 on the IS 1002 sequence, respectively, was performed with BamHIand SphI-digested and religated B. parapertussis chromosomal DNA to determine the termini of the element. Inverse PCR was performed as described previously (27). To sequence the IS1002 homolog of $B$. pertussis, which was harbored on cosmid p11-11 (19), the appropriate Sall fragments and the HpaIClal fragment were subcloned.

PCR detection of IS elements. The Bordetella strains were screened for the presence of IS elements by PCR, using primers based on the DNA sequences of IS1001 and IS1002a. The following primers were used: for IS1001, primers A and $\mathrm{Z}$, which are described above; and for IS1002, primers P3 $\left(5^{\prime}\right.$-TCCCAACTC CACGCACACCG-3') and H2 (5'-AACAACCATAAGCATGCGCG-3'), cor- responding to positions 797 to 777 and 93 to 113 on the sequence of IS1002a, respectively (Fig. 2). To isolate DNA, bacterial cells were scraped from agar plates and suspended in a physiological salt solution supplemented with $1 \mathrm{mM}$ EDTA. The preparations were diluted to an optical density at $600 \mathrm{~nm}$ of $2 \times$ $10^{-5}$ and boiled for $10 \mathrm{~min}$ to release the DNA, and $5 \mu \mathrm{l}$ of each suspension was used in a PCR. The PCR conditions used have been described previously (26). The reaction mixtures were preheated at $95^{\circ} \mathrm{C}$ for $3 \mathrm{~min}$. A total of 33 amplification cycles consisting of $1 \mathrm{~min}$ at $95^{\circ} \mathrm{C}, 1 \mathrm{~min}$ at $54^{\circ} \mathrm{C}$, and $1 \mathrm{~min}$ at $72^{\circ} \mathrm{C}$ were performed with a DNA thermocycler (Perkin-Elmer Cetus); this was followed by incubation at $5 \mathrm{~min}$ at $72^{\circ} \mathrm{C}$ to complete the final polymerase reaction.

DNA sequence analysis. The sequences of 420-bp DNA fragments of IS1001 amplified by primers $\mathrm{A}$ and $\mathrm{Z}$ were compared by using PCR products derived from the following strains: one Dutch $B$. parapertussis isolate obtained from a human, two B. parapertussis ovine isolates (from Scotland and New Zealand), three $B$. bronchiseptica isolates obtained from pigs (from The Netherlands, the United States, and Australia), and two isolates obtained from rabbits (from Denmark and Switzerland). The sequences of the PCR fragments were determined as described by Craxton (7) by using with dye-labeled dideoxynucleotides, primers $\mathrm{A}$ and $\mathrm{Z}$, and a model 370 automated sequencer (Applied Biosystems).

The sequences of plasmid clones containing IS1002 sequences were determined in both directions by using Taq polymerase (Promega), dye-labeled M13 primers or dye-labeled dideoxynucleotides, and primers P3, HG1, H, HG2, C, and $\mathrm{H} 2$ as described above. To investigate whether IS 481 had been inserted into IS1002, the sequence of the downstream flanking region of IS481 was determined by using primer S23 (5'-GGCGCGCTGTACCCATCTCC-3'), corresponding to positions 989 to 1009 on the IS481 sequence (19). Searches for sequence similarity were carried out with the BLAST algorithm (1).

Nucleotide sequence accession number. The IS1002 sequence has been deposited in the EMBL databank under accession number Z54268.

\section{RESULTS}

IS1001-associated RFLP analysis of $B$. parapertussis and $B$. bronchiseptica strains. In a previous study (27) we found that 12 human $B$. parapertussis strains isolated in The Netherlands produced a single IS1001 RFLP pattern, pattern X1 (Fig. 3A, lane 1 ). In this study we investigated 19 human $B$. parapertussis isolates obtained from various geographic areas (Finland, Germany, New Zealand, United Kingdom, Japan, and the United States). Again, most of the isolates produced the same fingerprint pattern, pattern X1 (Table 1 and Fig. 3A). Two slightly different fingerprint patterns were produced by a Finnish isolate and an American isolate (pattern X2) (Fig. 3A, lane 2) and by a German isolate (pattern X3) (Fig. 3A, lane 3).

To determine the genetic relationship of $B$. parapertussis isolates obtained from sheep with isolates obtained from humans, we subjected $22 B$. parapertussis strains obtained from sheep to Southern blotting and hybridization with IS1001 as the probe. The IS element was found in all of these strains. Although the number of IS1001 copies found in the sheep strains was similar to the number of copies found in the strains obtained from humans ( 20 to 23 copies), only a limited number of common hybridizing $S p h \mathrm{I}$ bands were found in the human and sheep isolates of B. parapertussis (approximately 5 of 20 bands). The IS1001 fingerprints of the sheep isolates were clearly distinct from those of the human isolates. Furthermore, the patterns of the sheep strains were more variable than the patterns of the human strains. The $B$. parapertussis strains isolated from sheep in Scotland produced three IS1001 band patterns (patterns Y2 to Y4) (Fig. 3A, lanes 5 to 7), while the strains from New Zealand produced only one pattern (pattern Y1) (Fig. 3A, lane 4). The latter pattern was very similar but not identical to the fingerprint pattern observed in the majority of the sheep strains from Scotland (pattern Y2).

A total of $41 \mathrm{~B}$. bronchiseptica strains were analyzed for the presence of IS1001. IS1001 was detected in 26 of these strains. The number of IS copies varied from one to seven, values which were much lower than the values found in $B$. parapertussis strains. Interestingly, there was an association between the presence of IS1001 and the host from which the B. bronchiseptica strains originated (Table 1). IS1001 was found in 15 of 16 pig strains and in 6 of 8 rabbit strains. However, IS1001 
TABLE 1. Bordetella strains used in this study

\begin{tabular}{|c|c|c|c|c|c|}
\hline Species & $\begin{array}{l}\text { No. of } \\
\text { strains }\end{array}$ & Host & $\begin{array}{l}\text { IS1001 RFLP } \\
\text { pattern }^{a}\end{array}$ & $\begin{array}{l}\text { IS1002 RFLP } \\
\text { pattern(s) }\end{array}$ & Geographic origin \\
\hline \multirow[t]{4}{*}{ B. pertussis } & 100 & Human & - & A4-A36 & The Netherlands \\
\hline & 1 & Human & - & A1 & Japan $^{c}$ \\
\hline & 1 & Human & - & A2 & United Kingdom ${ }^{d}$ \\
\hline & 1 & Human & - & $\mathrm{A} 3$ & United States \\
\hline \multirow[t]{14}{*}{ B. parapertussis } & 12 & Human & $\mathrm{X} 1$ & B1 (1) or ND (11) & The Netherlands \\
\hline & 4 & Human & $\mathrm{X} 1$ & B1 (2) or ND (2) & Finland \\
\hline & 2 & Human & $\mathrm{X} 1$ & B1 & Germany \\
\hline & 3 & Human & $\mathrm{X} 1$ & B1 (2) or ND (1) & New Zealand \\
\hline & 2 & Human & $\mathrm{X} 1$ & B1 or ND & United Kingdom \\
\hline & 4 & Human & $\mathrm{X} 1$ & ND & Japan \\
\hline & 1 & Human & $\mathrm{X} 1$ & B1 & United States \\
\hline & 1 & Human & $\mathrm{X} 2$ & B1 & Finland \\
\hline & 1 & Human & $\mathrm{X} 2$ & B1 & United States \\
\hline & 1 & Human & $\mathrm{X} 3$ & B1 & Germany \\
\hline & 5 & Sheep & Y1 & - & New Zealand \\
\hline & 15 & Sheep & $\mathrm{Y} 2$ & - & Scotland \\
\hline & 1 & Sheep & $\mathrm{Y} 3$ & - & Scotland \\
\hline & 1 & Sheep & Y4 & - & Scotland \\
\hline \multirow[t]{33}{*}{ B. bronchiseptica } & 1 & Pig & - & - & United Kingdom \\
\hline & 1 & Pig & $\mathrm{R} 1$ & - & The Netherlands \\
\hline & 1 & Pig & $\mathrm{R} 3$ & - & Australia \\
\hline & 1 & Pig & R4 & - & South Africa \\
\hline & 1 & Pig & $\mathrm{R} 5$ & - & USSR \\
\hline & 1 & Pig & R7 & - & Australia \\
\hline & 2 & Pig & R10 & - & The Netherlands \\
\hline & 1 & Pig & $\mathrm{R} 12$ & - & United States \\
\hline & 1 & Pig & $\mathrm{R} 13$ & - & The Netherlands \\
\hline & 1 & Pig & R14 & - & The Netherlands \\
\hline & 1 & Pig & R15 & - & The Netherlands \\
\hline & 1 & Pig & R16 & - & The Netherlands \\
\hline & 2 & Pig & R17 & - & South Africa \\
\hline & 1 & Pig & R18 & - & USSR \\
\hline & 4 & Dog & - & - & The Netherlands \\
\hline & 1 & Dog & - & - & South Africa \\
\hline & 1 & Dog & R9 & - & The Netherlands \\
\hline & 2 & Cat & - & - & The Netherlands \\
\hline & 1 & Cat & R2 & - & The Netherlands \\
\hline & 1 & Rabbit & - & - & The Netherlands \\
\hline & 1 & Rabbit & - & - & United States \\
\hline & 1 & Rabbit & R11 & - & Switzerland \\
\hline & 2 & Rabbit & R17 & - & South Africa \\
\hline & 1 & Rabbit & $\mathrm{R} 19$ & - & Denmark \\
\hline & 1 & Rabbit & $\mathrm{R} 20$ & - & The Netherlands \\
\hline & 1 & Rabbit & R21 & - & United States \\
\hline & 1 & Horse & - & $-f$ & Denmark \\
\hline & 1 & Cow & R6 & - & Ireland \\
\hline & 1 & Mouse & $\mathrm{R} 8$ & - & United States \\
\hline & 1 & Unknown & $\mathrm{R} 17$ & - & South Africa \\
\hline & 1 & Koala & - & + & Australia \\
\hline & 2 & Human & - & - & The Netherlands \\
\hline & 1 & Human & - & - & Germany \\
\hline
\end{tabular}

$a_{-}$, strain(s) do(es) not contain IS1001.

$b_{-}$, strain(s) do(es) not contain IS1002; +, strain contains IS1002; ND, not determined. The numbers in parentheses are numbers of strains.

${ }^{c}$ Data from reference 13.

${ }^{d}$ Data from reference 17.

${ }^{e}$ Data from reference 14.

${ }^{f}$ This strain contains IS 481 .

was present in only 2 of 9 strains obtained from dogs and cats. The IS1001 fingerprints of $B$. bronchiseptica strains were clearly distinct from the $B$. parapertussis patterns, and more variation was observed among the $B$. bronchiseptica strains than among the $B$. parapertussis strains. A total of 21 different fingerprint patterns were observed among the strains analyzed, a selection of which is shown in Fig. 3A. The human and ovine B. parapertussis strains shared a number of hybridizing SphI bands with a $B$. bronchiseptica strain obtained from a pig (Fig. 3A, lane 8, 13-, 7.4-, 5.2-, 4.3-, and 2.5-kb bands), suggesting that common sites for IS1001 insertion were present. Most of these bands were also observed in other B. bronchiseptica strains which we analyzed (Fig. 3A). When chromosomal DNA was digested with SmaI to generate IS1001 fingerprints, 4.8-, $3.5-, 3.3-$, and 2.6-kb bands were found in both the pig $B$. bronchiseptica strain and $B$. parapertussis strains isolated from 


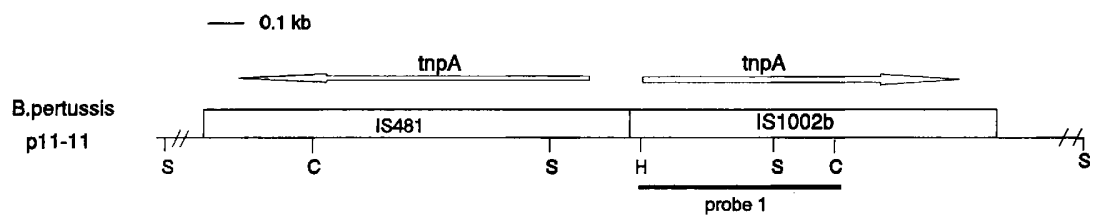

\section{B.parapertussis pRPP30}

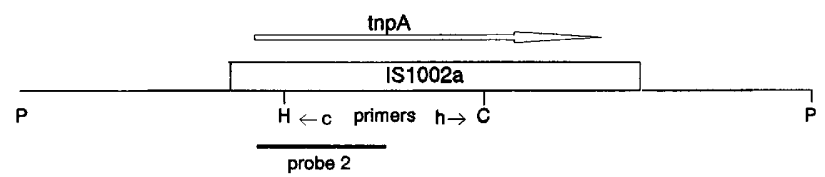

FIG. 1. Genetic and physical maps of the chromosomal regions of $B$. pertussis and B. parapertussis harboring IS481 and/or IS1002 and probes and primers used. The locations of the putative transposase genes $(\operatorname{tnp} A)$ are indicated by large arrows. Small arrows $\mathrm{c}$ and $\mathrm{h}$ indicate the positions of the primers used to clone the IS1002-flanking regions of B. parapertussis by inverse PCR. Probes 1 and 2 were generated by PCR and used to isolate IS1002 from B. parapertussis and for DNA fingerprinting, respectively. Abbreviations: C, ClaI; S, SalI; H, HpaI; P, PstI.

sheep and humans (Fig. 3B). In addition, a 1.4-kb band was found in only the $B$. bronchiseptica strain and human $B$. parapertussis strains (Fig. 3B). The occurrence of shared bands suggests that the three (sub)species descended from an ancestor which already contained IS1001. The distinct RFLP patterns of the human and ovine $B$. parapertussis strains indicate that these organisms constitute two separate populations. Furthermore, the human $B$. parapertussis population appears to be very homogeneous, whereas the ovine $B$. parapertussis strains are genetically more heterogeneous. The $B$. bronchiseptica strains exhibited the highest degree of IS1001-associated DNA polymorphism.

Comparison of IS1001 sequences of different $B$. parapertussis and $B$. bronchiseptica strains. To investigate the divergence of IS1001 element sequences in different Bordetella species, we determined the DNA sequences of internal 420-bp PCR fragments from three $B$. parapertussis strains (one human strain and two ovine strains) and five $B$. bronchiseptica strains (three strains from pigs and two strains from rabbits). The results of this analysis are shown in Figure 4. The maximum level of sequence divergence was $1.5 \%$. The DNA sequence of the 420 -bp IS 1001 fragment of the human $B$. parapertussis strain was identical to the sequence of one of the $B$. bronchiseptica pig strains and differed from the sequences of the two other pig strains in only one residue. The DNA sequences of the two ovine $B$. parapertussis strains were identical and differed from the sequences of the human strain in four residues. The IS1001 sequence of one of the two rabbit $B$. bronchiseptica strains was the most divergent (Fig. 4).

Characterization of a novel IS element designated IS1002. As mentioned above, human $B$. parapertussis strains harbor a second, uncharacterized repetitive DNA element, which originally was discovered in $B$. pertussis (19). This element was detected previously on a cosmid (p11-11) derived from $B$. pertussis, and it was found to be located adjacent to the IS481 sequence on this cosmid (19). To characterize this repetitive DNA element, we cloned a homologous sequence from $B$. parapertussis. To do this, a probe containing the $B$. pertussis repetitive DNA (Fig. 1, probe 1) was derived from p11-11 and used to select its homologue by hybridization with a pool of cloned B. parapertussis PstI fragments. One of the hybridizing plasmids obtained was designated pRPP30, and this plasmid was found to contain a $1.8-\mathrm{kb}$ Pst I fragment. This fragment was sequenced. To identify the termini of the repetitive sequence, the flanking DNA of this repeat was cloned by inverse PCR with primers $h$ and $\mathrm{c}$ (Fig. 1) on restricted, religated $B$. parapertussis chromosomal DNA. The sequences of the PCR frag- ments containing different ends of the repeat allowed us to delineate the DNA element.

The repetitive DNA sequence had all of the characteristic features of an IS element (see below). We designated this IS element IS1002. The copies of IS1002 derived from $B$. parapertussis and $B$. pertussis are referred to in this paper as IS1002 $a$ and IS $1002 b$, respectively. IS $1002 a$ was 1,040 bp long and had a $\mathrm{G}+\mathrm{C}$ content of $63.9 \mathrm{~mol} \%$ and 29-bp inverted terminal repeats (Fig. 2). The junctions at the site of insertion showed no target duplication.

We also determined the sequence of IS1002b, which is located on cosmid p11-11. This IS element was truncated compared with IS1002a. The 5' 127 bp were missing in IS1002b, and because IS 481 sequences directly bordered the truncated sequence (Fig. 1 and 2), it is conceivable that the truncation was the result of transposition of IS481 into this copy of IS1002. The sequence downstream of IS481, however, exhibited no homology to IS 1002 (data not shown).

The $B$. pertussis and B. parapertussis copies of IS1002 exhibited a high level of DNA sequence identity (93\%). A search of the GenBank and EMBL databases revealed that the most similar sequence was the IS481 sequence (level of sequence identity, $61.5 \%$ ). The inverted terminal repeats of IS1002 and IS481 were almost identical. The major open reading frame (ORF) of IS1002a, comprising 214 amino acids, exhibited a level of sequence identity of $29.4 \%$ with the $129 \mathrm{~N}$-terminal amino acids of the putative transposase encoded by IS481. The results of a comparison of the ORFs and DNA sequences of IS1002 $a$ and IS1002 $b$ suggested that a deletion of a single base had occurred in IS1002a at position 474 (Fig. 2), resulting in a frameshift. When the frameshift was corrected, an ORF consisting of 316 amino acid residues was obtained in IS1002a, and the level of amino acid sequence identity with the putative transposase encoded by IS 481 was $48 \%$. Significant levels of sequence identity were also found with IS elements found in $E$. coli, Xanthomonas campestris, and Mycobacterium intracellulare (data not shown).

Host range of IS1002 in $B$. pertussis, $B$. parapertussis, and $B$. bronchiseptica strains. The host range of IS1002 was investigated by PCR by using IS1002-specific primers. IS1002 was detected in all $100 \mathrm{~B}$. pertussis strains investigated and all $31 \mathrm{~B}$. parapertussis human strains investigated. However, it was absent from all $22 B$. parapertussis ovine strains tested and was found in only 1 of $100 \mathrm{~B}$. bronchiseptica strains tested (Table 1).

IS1002-associated RFLP analysis of $B$. pertussis, $B$. parapertussis, and $B$. bronchiseptica strains. Southern blot analyses of SmaI-restricted chromosomal DNAs of different Bordetella 


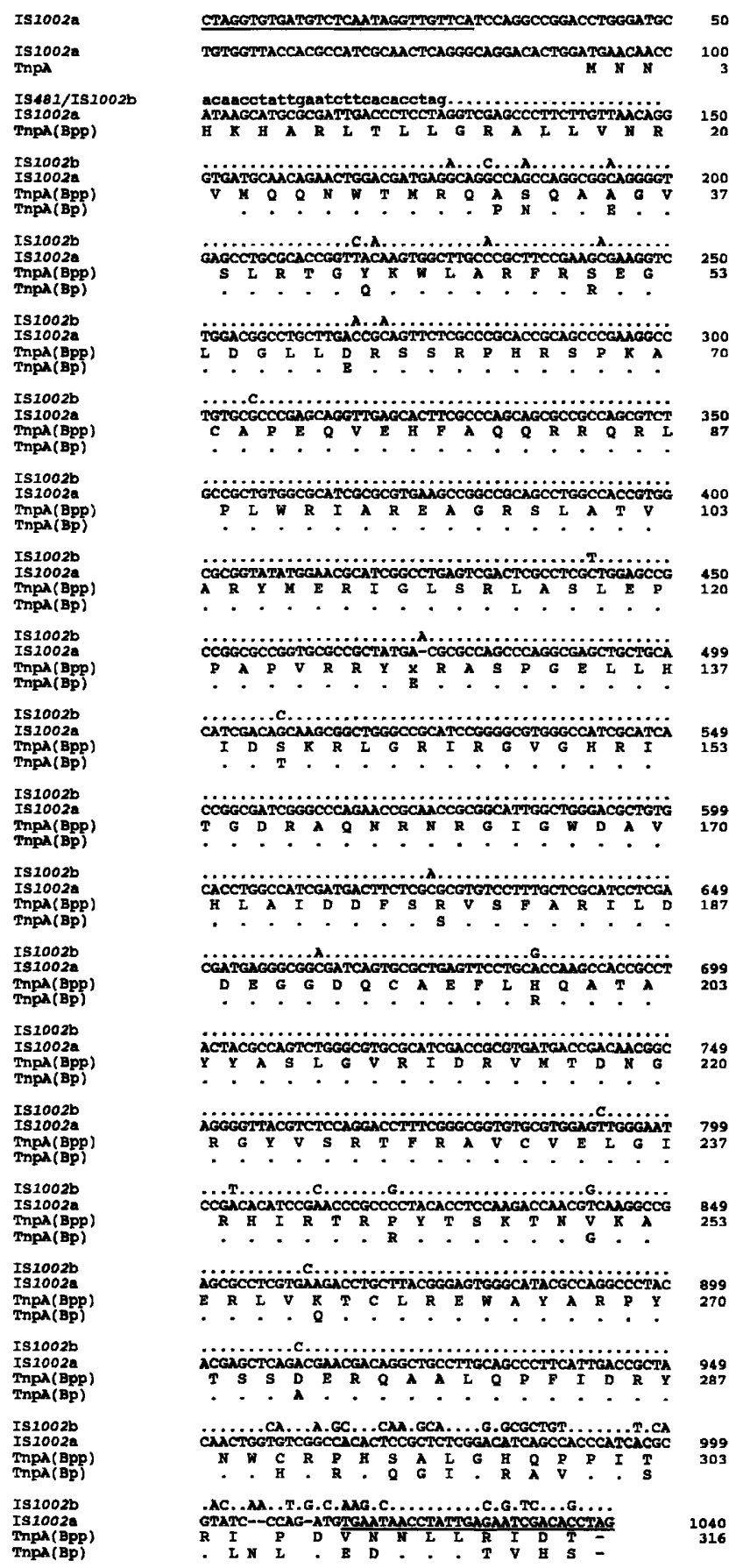

FIG. 2. DNA sequences of IS1002a from $B$. parapertussis and IS1002 $b$ from $B$. pertussis. The inverted repeats are underlined. The derived amino acid sequence of the putative transposase is also shown. The dash at amino acid residue 128 indicates a gap introduced to restore the ORF. All other dashes in the DNA sequence were introduced to maximize alignment of the sequences. Bases of IS $1002 b$ identical to IS1002a bases and amino acid residues identical to IS $1002 a$ encoded ORF residues are indicated by dots above and below the sequence of IS $1002 a$, respectively. The left inverted repeat of IS 481 , interrupting IS $1002 b$, is shown in lowercase letters. The numbers of bases and amino acid residues are indicated on the right. Abbreviations: Bpp., B. parapertussis; Bp., B. pertussis.

strains were performed by using an internal fragment of IS1002a as the probe (Fig. 1, probe 2). Four to seven hybridizing SmaI bands were observed in the patterns of the three $B$. pertussis strains investigated, and all three patterns were dif- ferent. The Southern blot analysis of 13 human B. parapertussis strains revealed nine hybridizing fragments, and all of the patterns were identical (Table 1 and Fig. 5). The single B. bronchiseptica strain containing IS1002 produced one hybridizing band. None of the $22 \mathrm{~B}$. parapertussis strains from sheep hybridized with IS1002 DNA (data not shown), confirming the PCR results described above.

\section{DISCUSSION}

In this study we exploited DNA polymorphism associated with repetitive DNA to study the population structure of the genus Bordetella. Previously, similar studies have been performed successfully with other bacteria, such as Mycobacterium tuberculosis (28). For a long time it was assumed that $B$. parapertussis is an obligate human pathogen that generally causes a milder form of whooping cough than $B$. pertussis. This view changed in 1987 when $B$. parapertussis was isolated from sheep (8). To investigate whether $B$. parapertussis may be transmitted from this animal reservoir and infect humans, we studied the population structure of $B$. parapertussis by using repetitive DNA.

We characterized a novel IS element, which was designated IS1002. IS1002 appeared to be closely related to another IS element, IS481, which is found in $B$. pertussis but not in $B$. parapertussis and in only 1 of the 41 strains of $B$. bronchiseptica investigated. These two elements exhibit $61.5 \%$ sequence identity and have almost identical terminal inverted repeats. IS1002 was found in all $B$. pertussis strains and all $B$. parapertussis strains isolated from humans. In contrast, none of the $B$. parapertussis strains from sheep investigated contained this element. The distribution of IS1002 suggests that the B. parapertussis strains isolated from sheep and humans represent two separate populations which have adapted to different hosts.

Another IS element, IS1001, has been found previously in human $B$. parapertussis isolates and in some $B$. bronchiseptica isolates (27). In this study, IS1001 was also found in all $B$. parapertussis isolates obtained from sheep. The IS1001-related DNA fingerprints of human and ovine strains were clearly distinct, providing additional evidence that the human and ovine $B$. parapertussis strains make up distinct populations. This is consistent with the observation of Porter et al. (24) that ovine $B$. parapertussis strains can be differentiated from human strains on the basis of biochemical characterization and MEE data. Therefore, we concluded that the reservoirs of $B$. parapertussis in sheep and humans constitute two different bacterial populations and that little or no transmission takes place between these two reservoirs.

We found that most human $B$. parapertussis strains produce a single IS1001 fingerprint pattern, pattern X1. Only three of the human strains investigated produced a slightly different pattern, which differed in the position of only one or two bands. When IS1002 was used to generate DNA fingerprints, only one fingerprint pattern was obtained for human $B$. parapertussis strains, whereas various patterns were obtained for $B$. pertussis strains. The results of these analyses indicate that the human $B$. parapertussis population is genetically very homogeneous and thus probably descended relatively recently from a single clone. This is consistent with the preliminary data of other workers. Pulsed-field gel electrophoresis of 10 human $B$. parapertussis strains isolated at different times or from various locations revealed three electrophoretic types (15), and an MEE analysis of 21 human $B$. parapertussis strains revealed only one electrophoretic type (23). Thus, the results obtained with two other approaches to differentiate strains also indicate that the human $B$. parapertussis population is highly clonal. 
A

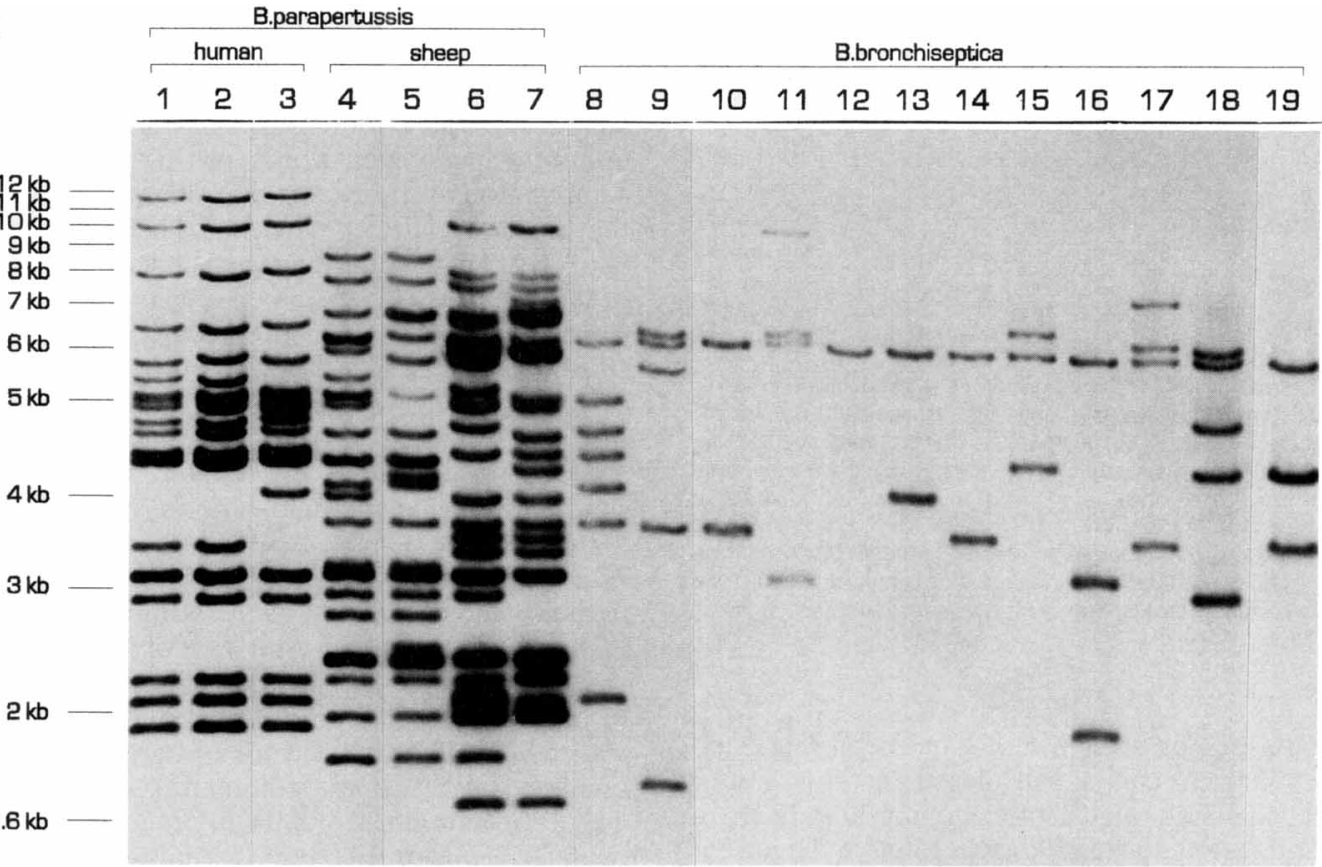

$\begin{array}{lllllllllllllllllll}X 1 & X 2 & X 3 & Y 1 & Y 2 & Y 3 & Y 4 & R 1 & R 2 & R 3 & R 4 & R 5 & R 6 & R 7 & R 8 & R 9 & R 10 & R 11 & R 12\end{array}$

B
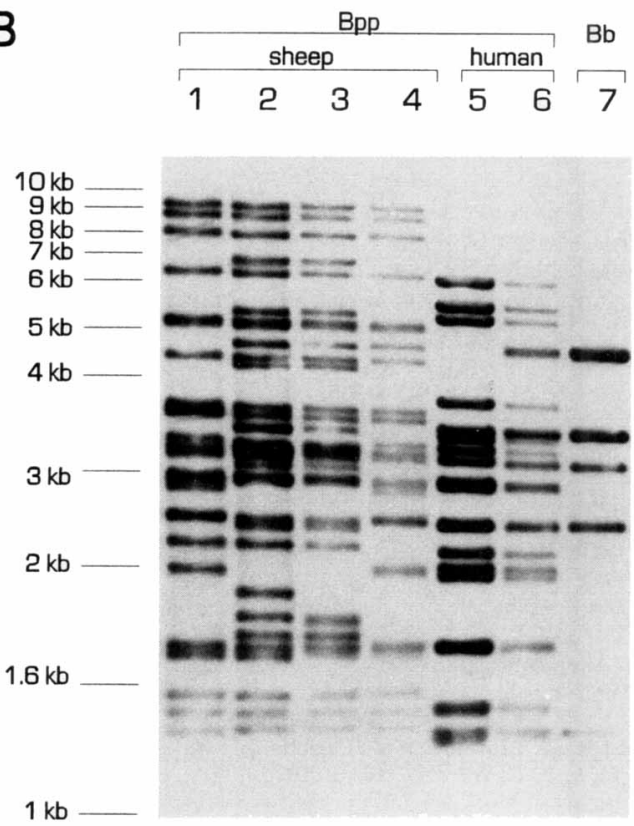

Populations of pathogenic bacteria are generally genetically more diverse (29) than the human $B$. parapertussis isolates observed in this study. Two alternative explanations could account for the genetic homogeneity of human $B$. parapertussis strains. First, a $B$. parapertussis clone may have escaped from the animal reservoir and become adapted to human hosts only recently. Consequently, not enough time has passed for divergence to have occurred. Second, the association of $B$. parapertussis with humans may be quite ancient, but a single clone may have expanded worldwide relatively recently; perhaps this clone acquired IS1002.
FIG. 3. IS1001-generated fingerprints of B. parapertussis and B. bronchiseptica strains. Chromosomal DNA was restricted with SphI (A) or SmaI (B), and an internal fragment from IS1001 was used as the probe. (A) Lanes 1 through 7, B. parapertussis strains (lane 1, human strain from The Netherlands; lane 2, human strain from Finland; lane 3, human strain from Germany; lane 4, sheep strain from New Zealand; lanes 5 through 7, sheep strains from Scotland); lanes 8 through $19, B$. bronchiseptica strains (lane 8 , pig strain from The Netherlands; lane 9, cat strain from The Netherlands; lane 10, pig strain from Australia; lane 11, pig strain from South Africa; lane 12, pig strain from the USSR; lane 13, cow strain from Ireland; lane 14, pig strain from Australia; lane 15, mouse strain from the United States; lane 16, dog strain from The Netherlands; lane 17, pig strain from The Netherlands; lane 18, rabbit strain from Switzerland; lane 19, pig strain from the United States). The difference in the fingerprint patterns in lanes 1 and 2 consists of the slightly lower mobility of the fifth band (from the top) in lane 2. (B) Lanes 1 through 3, sheep strains from Scotland; lane 4, sheep strain from New Zealand; lane 5, human strain from The Netherlands; lane 6, human strain from the United States; lane 7, B. bronchiseptica strain from a pig in The Netherlands. All $B$. parapertussis strains analyzed in this study produced hybridization patterns identical to one of the patterns shown, and the RFLP patterns are shown in Table 1. The designations of the RFLP patterns are indicated at the bottom. The sizes of 1-kb marker DNA fragments are indicated on the left.

As $B$. pertussis is found only in humans, the most parsimonious explanation for the observed distribution of IS1002 seems to be that relatively recently a strain of $B$. parapertussis in a human acquired IS1002 from a coinfecting $B$. pertussis strain. IS1002 was found in only one $B$. bronchiseptica strain isolated from a koala. This strain also may have acquired IS1002 by horizontal transfer.

In contrast to the IS1002 RFLP patterns of $B$. parapertussis strains from humans, the IS1002 band patterns of $B$. pertussis strains were quite polymorphic. This suggests that IS1002 may be a useful tool for studying the epidemiology of whooping cough. At this time, highly discriminative tools for tracing the transmission routes of $B$. pertussis in addition to pulsed-field gel electrophoresis are very limited. We are now using IS1002 to study the epidemiology of $B$. pertussis by performing experiments similar to experiments done with $M$. tuberculosis (28).

On the basis of the phylogenetic relationships in the genus 


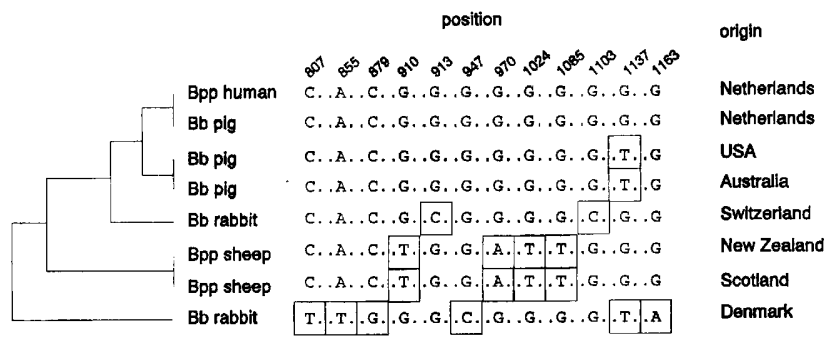

FIG. 4. Comparison of the DNA sequences of PCR products internal to IS1001 derived from $B$. parapertussis human and ovine isolates and B. bronchiseptica pig and rabbit isolates. The PCR fragment was $420 \mathrm{bp}$ long, ranging from position 764 to position 1184 on the sequence of IS1001 as described previously (27). The positions at which base substitutions were found are indicated above the sequence, and bases that differed from IS1001 bases are enclosed in boxes. The host strains are indicated on the left. The origins of the strains tested are indicated on the right. The dendrogram was generated by comparing the 420 -bp sequences by neighbor joining. Abbreviations: Bpp, B. parapertussis; Bb, B. bronchiseptica.

Bordetella, as established by using MEE, it has been suggested that $B$. parapertussis strains can be considered derivatives of $B$. bronchiseptica that have adapted to the human host (23). In this context it is interesting that IS1001 was found to be absent from $B$. pertussis, but present in all human and ovine $B$. parapertussis strains and in most $B$. bronchiseptica strains used in this study. Most of the $B$. bronchiseptica strains which contained IS1001 were obtained from pigs or rabbits, suggesting that these organisms represent distinct groups that became specialized and infect particular animals. This suggestion is supported by the results of previous MEE analyses of the $B$. bronchiseptica pig strains, most of which have been shown to belong to a single electrophoretic type (22). The IS1001 band patterns of $B$. bronchiseptica strains were more diverse than the $B$. parapertussis patterns. This finding supports the hypothesis that $B$. bronchiseptica represents the oldest phylogenetic branch in the genus Bordetella (23). The ovine B. parapertussis strains exhibited more variation in their IS1001 band patterns than the human strains. The greater polymorphism in the ovine

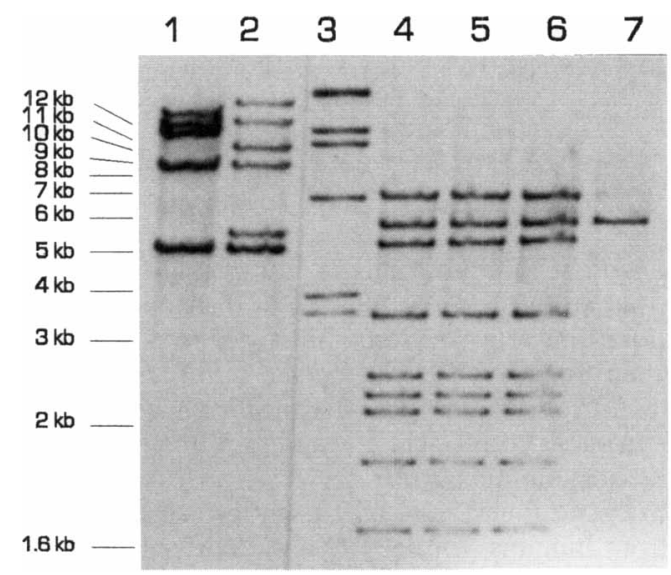

FIG. 5. IS1002-generated fingerprints of B. pertussis, human B. parapertussis, and B. bronchiseptica strains. Chromosomal DNAs were restricted with SmaI, and an internal fragment derived from IS1002 was used as the probe (Fig. 1, probe 2). Lane 1, B. pertussis Tohama (24); lane 2, B. pertussis Wellcome 28 (25); lane $3, B$. pertussis 18323 (28); lane $4, B$. parapertussis human strain from The Netherlands; lane $5, B$. parapertussis human strain from Finland; lane $6, B$. parapertussis human strain from Germany; lane $7, B$. bronchiseptica koala strain from Australia. The sizes of 1-kb marker DNA fragments are indicated on the left. strains suggests that the human $B$. parapertussis strains evolved more recently than the ovine strains.

Arber (2) and Blot (4) have reviewed the putative role of IS elements in the evolution of microbial species and their adaptation to changing environments. It may well be that a specific combination of insertions of IS elements leads to a phenotype that is beneficial in a particular host. Thus, IS1001-mediated DNA rearrangements may have played a role in the adaptation of $B$. parapertussis to either sheep or humans. The strains isolated from these two hosts were found to be distinct, but closely related in terms of copy number and a limited number of common sites of insertion of IS1001, suggesting that these organisms descended from a common ancestor, possibly a $B$. bronchiseptica strain in the animal reservoir, and that once they were in the ovine and human hosts, selection for strains with a higher IS copy number may have occurred. The results of our comparison of DNA sequences internal to IS1001 in B. parapertussis and $B$. bronchiseptica strains from humans and animals showed that the overall level of homology is very high and that the human $B$. parapertussis strains are more closely related to $B$. bronchiseptica strains from pigs than the ovine $B$. parapertussis strains are. The dendrogram based on IS1001 sequences suggests that the human $B$. parapertussis strains share a more recent ancestor with $B$. bronchiseptica strains than the ovine $B$. parapertussis strains. Thus, the results of both comparisons of IS1001-associated RFLP and comparisons of IS1001 sequences suggest that the ovine and human $B$. parapertussis strains evolved independently from $B$. bronchiseptica strains.

\section{ACKNOWLEDGMENTS}

We thank J. Mertsola, C. H. Wirsing von König, P. Short, T. Aoyama, B. W. Manktelow, J. Porter, and W. Gaastra for providing strains and Jan van Embden for critically reading the manuscript.

This work was supported in part by Het Praeventiefonds.

\section{REFERENCES}

1. Altschul, S. F, W. Gish, W. Miller, E. W. Myers, and D. J. Lipman. 1990 Basic local alignment search tool. J. Mol. Biol. 215:403-410.

2. Arber, W. 1990. Mechanisms in microbial evolution. J. Struct. Biol. 104:107-11.

3. Ausubel, F. M., R. Brent, R. E. Kingston, D. D. Moore, J. G. Smith, J. G. Seidman, and J. Doly. 1987. Current protocols in molecular biology. John Wiley \& Sons, New York.

4. Blot, M. 1994. Transposable elements and adaptation of host bacteria. Genetics 93:5-12.

5. Chen, W., M. R. Alley, B. W. Manktelow, D. Hopcroft, and R. Bennett. 1988 Pneumonia in lambs inoculated with Bordetella parapertussis: bronchoalveolar lavage and ultrastructural studies. Vet. Pathol. 25:297-303.

6. Coote, J. G., and R. M. Brownlie. 1988. Genetics of virulence of Bordetella pertussis, p. 39-74. In A. C. Wardlaw, and R. Parton (ed.), Pathogenesis and immunity in pertussis. John Wiley and Sons, New York.

7. Craxton, M. 1991. Linear amplification sequencing; a powerful method for sequencing DNA. Methods Enzymol. 2:1-7.

8. Cullinane, L. C., M. R. Alley, R. B. Marshall, and B. W. Manktelow. 1987. B. parapertussis from lambs. N.Z. Vet. J. 35:175.

9. Dente, L., M. Sollazo, C. Baldari, G. Cesareni, and R. Cortese. 1985. The pEMBL family of single strand vectors, p. 101-107. In D. M. Glover (ed.) DNA cloning: a practical approach, vol. I. IRL Press, Oxford.

10. Flosdorf, E. W., A. Bondi, H. Felton, and A. C. McGuinness. 1942. Studies with Haemophilus pertussis. X. Comparative antigenic analysis of Bacillus parapertussis and Haemophilus pertussis, phase I, with consideration of clinical significance. J. Pediatr. 21:625-634.

11. Heininger, U., K. Stehr, S. Schmitt-Grohe, C. Lorenz, R. Rost, P. D. Christenson, M. Uberall, and J. D. Cherry. 1994. Clinical characteristics of illness caused by Bordetella parapertussis compared with illness caused by Bordetella pertussis. Pediatr. Infect. Dis. J. 13:306-309.

12. Hewlett, E. L. 1990. Bordetella species, p. 1756-1762. In G. L. Mandell, R. G. Douglas, and J. E. Bennet (ed.), Principles and practice of infectious diseases. Churchill Livingstone, Inc., New York.

13. Kasuga, T., Y. Nakase, K. Ukishima, and K. Takatsu. 1953. Studies on 
Haemophilus pertussis. Kitasato Arch. Exp. Med. 27:21-28.

14. Kendrick, P. L., G. Eldering, M. K. Dixon, and J. Misner. 1947. Mouse protection tests in the study of pertussis vaccines: a comparative series using intracerebral route of challenge. Am. J. Public Health 37:803-810.

15. Khattak, M. N., and R. C. Matthews. 1993. Genetic relatedness of Bordetella species as determined by macrorestriction digests resolved by pulsed-field gel electrophoresis. Int. J. Syst. Bacteriol. 43:659-664.

16. Linneman, C. C., and E. B. Perry. 1977. Bordetella parapertussis: recent experience and a review of the literature. Am. J. Dis. Child. 13:560-563.

17. Livey, I., C. J. Duggleby, and A. Robinson. 1987. Cloning and nucleotide sequence analysis of the serotype 2 fimbrial subunit gene of Bordetella pertussis. Mol. Microbiol. 1:203-209.

18. Maniatis, T., E. F. Fritsch, and J. Sambrook. 1982. Molecular cloning: a laboratory manual. Cold Spring Harbor Laboratory, Cold Spring Harbor, N.Y.

19. McLafferty, M. A., D. R. Harcus, and E. L. Hewlett. 1988. Nucleotide sequence and characterization of a repetitive DNA element from the genome of Bordetella pertussis with characteristics of an insertion sequence. J. Gen. Microbiol. 134:2297-2306.

20. Mertsola, J. 1985. Mixed outbreak of Bordetella pertussis and Bordetella parapertussis infection in Finland. Eur. J. Clin. Microbiol. 4:123-128.

21. Miller, J. J., Jr., T. M. Saito, and R. J. Silverberg. 1941. Parapertussis: clinical and serologic observations. J. Pediatr. 19:229-240.

22. Musser, J. M., D. A. Bemis, H. Ishikawa, and R. K. Selander. 1987. Clonal diversity and host distribution in Bordetella bronchiseptica. J. Bacteriol. 169: 2793-2803.

23. Musser, J. M., E. L. Hewlett, M. S. Peppler, and R. K. Selander. 1986.
Genetic diversity and relationships in populations of Bordetella spp. J. Bacteriol. 166:230-237.

24. Porter, J. F., K. Connor, and W. Donachie. 1994. Isolation and characterization of Bordetella parapertussis-like bacteria from ovine lungs. Microbiology 140:255-261.

25. Preston, N. W. 1988. Pertussis today, p. 1-18. In A. C. Wardlaw and R. Parton (ed.), Pathogenesis and immunity in pertussis. John Wiley and Sons, New York.

26. van der Zee, A., C. Agterberg, M. Peeters, J. Schellekens, and F. R. Mooi. 1993. A PCR assay for whooping cough: simultaneous detection and discrimination of Bordetella pertussis and Bordetella parapertussis. J. Clin. Microbiol. 31:2134-2140.

27. van der Zee, A., C. Agterberg, M. van Agterveld, M. Peeters, and F. R. Mooi 1993. Characterization of IS1001, an insertion sequence element of Bordetella parapertussis. J. Bacteriol. 175:141-147.

28. Van Embden, J. D., M. D. Cave, J. T. Crawford, J. W. Dale, K. D. Eisenach, B. Gicquel, P. Hermans, C. Martin, R. McAdam, T. M. Shinnick, et al. 1993. Strain identification of Mycobacterium tuberculosis by DNA fingerprinting: recommendations for a standardized methodology. J. Clin. Microbiol. 31: 406-409.

29. Whittam, T. S. 1995. Genetic population structure and pathogenicity in enteric bacteria, p. 217-246. In S. Baumberg, J. Young, E. Wellington, and J. Saunders (ed.), Population genetics of bacteria. Cambridge University Press, Cambridge.

30. Wirsing von König, C. H., and H. Finger. 1994. Role of pertussis toxin in causing symptoms of Bordetella parapertussis infection. Eur. J. Clin. Microbiol. Infect. Dis. 13:455-458. 